\title{
An Empirical Approach for the Evaluation of Haptic Algorithms
}

Chris Raymaekers, Joan De Boeck, Karin Coninx Limburgs Universitair Centrum

Expertise centre for Digital Media (EDM)

Universitaire Campus, B-3590 Diepenbeek, Belgium

\{chris.raymaekers, joan.deboeck, karin.coninx\}@luc.ac.be

The number of haptic algorithms has been growing over the past few years. However, little research has been performed in evaluating these algorithms. This poster provides a discussion of how force-feedback algorithms can be empirically evaluated for correctness and performance. More information can be found in our paper in the conference proceedings.
Existing Performance Comparison Methodologies

- Theoretical comparison: calculation of time complexity

- Overload test: try to simulate as much triangles/objects as possible, until the haptic loop breaks down.

- Haptic Load Tool: compare the haptic load, read from a graphical tool

\section{Problem With Current Methods}

- Algorithms are compared with different data

- No exact numerical data to compare

- Tests often unintentionally measure scene graph overhead

\section{Our Approach to Compare Haptic Algorithms}
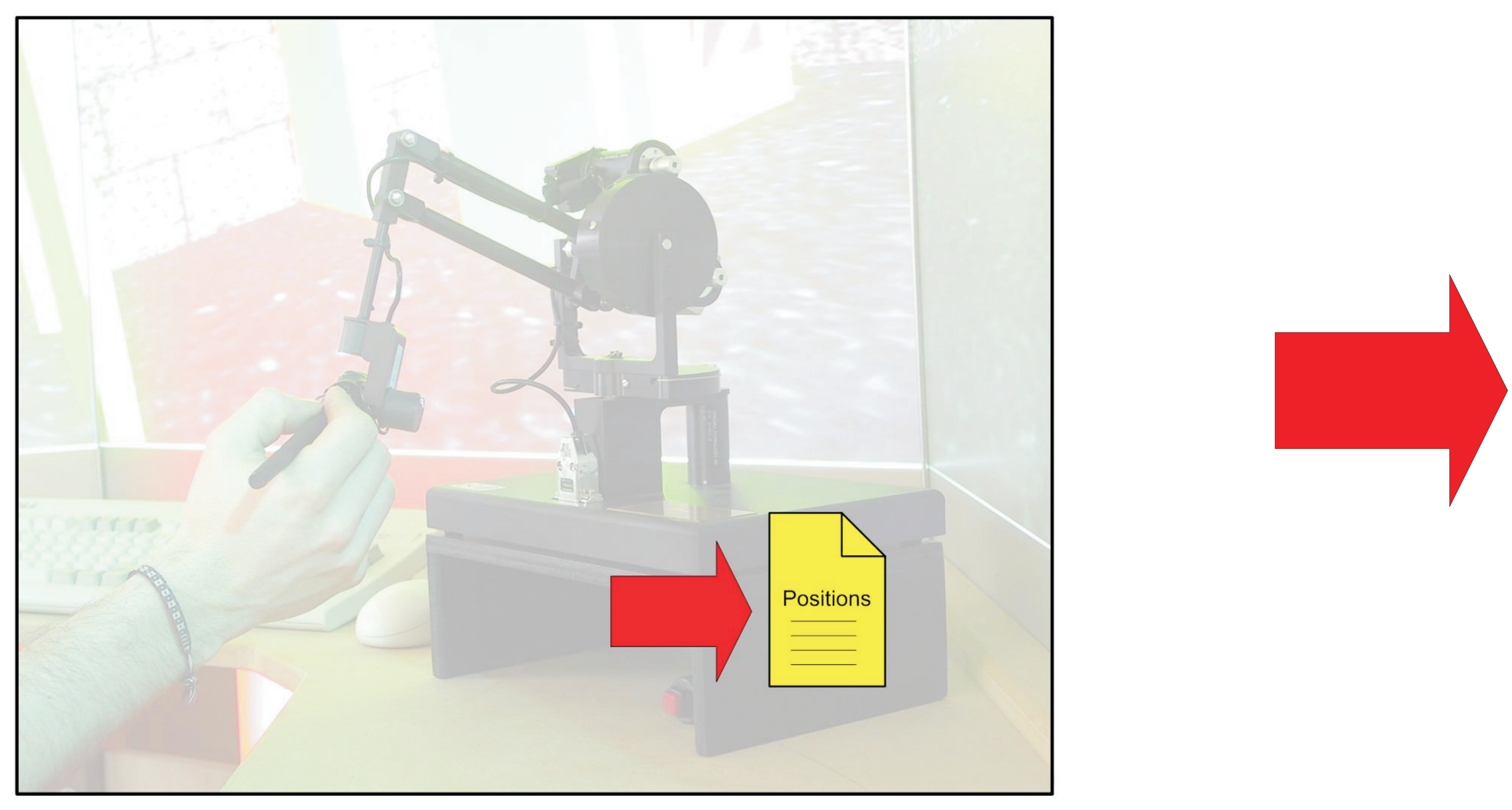

$$
\begin{aligned}
& \text { In a first step, a given number of users explores a given number } \\
& \text { of objects for a certain time using a given reference algorithm. } \\
& \text { Each execution of the haptic loop, the pointer's position and } \\
& \text { velocity is stored to a file. The results (contact point and } \\
& \text { rendered force) of the algorithm are saved to a result file. }
\end{aligned}
$$
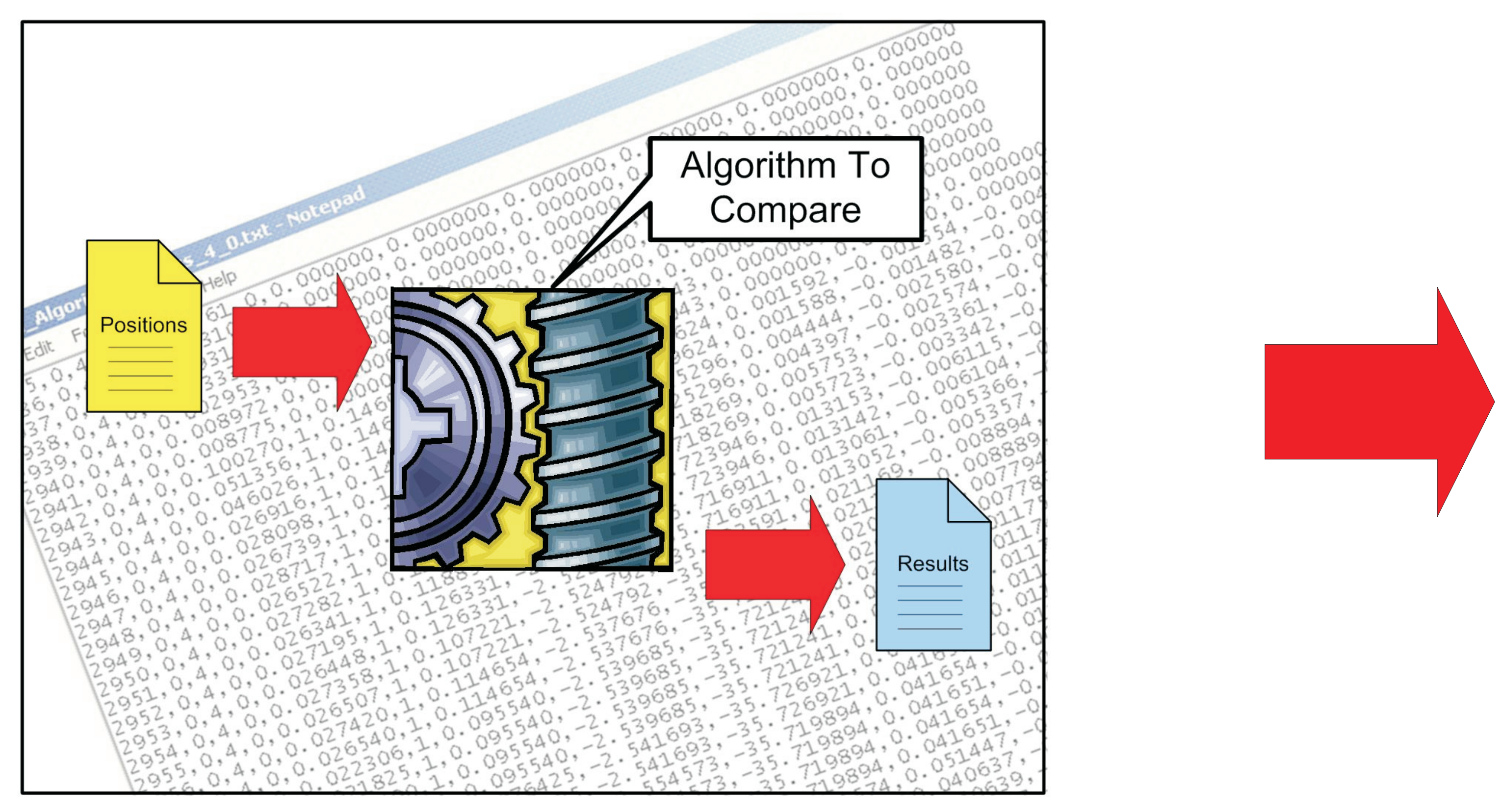

Apply Data to Other Algorithms

The original position file is processed by the new algorithm. The haptic algorithm's result (contact point and rendered force) are stored in a result file.
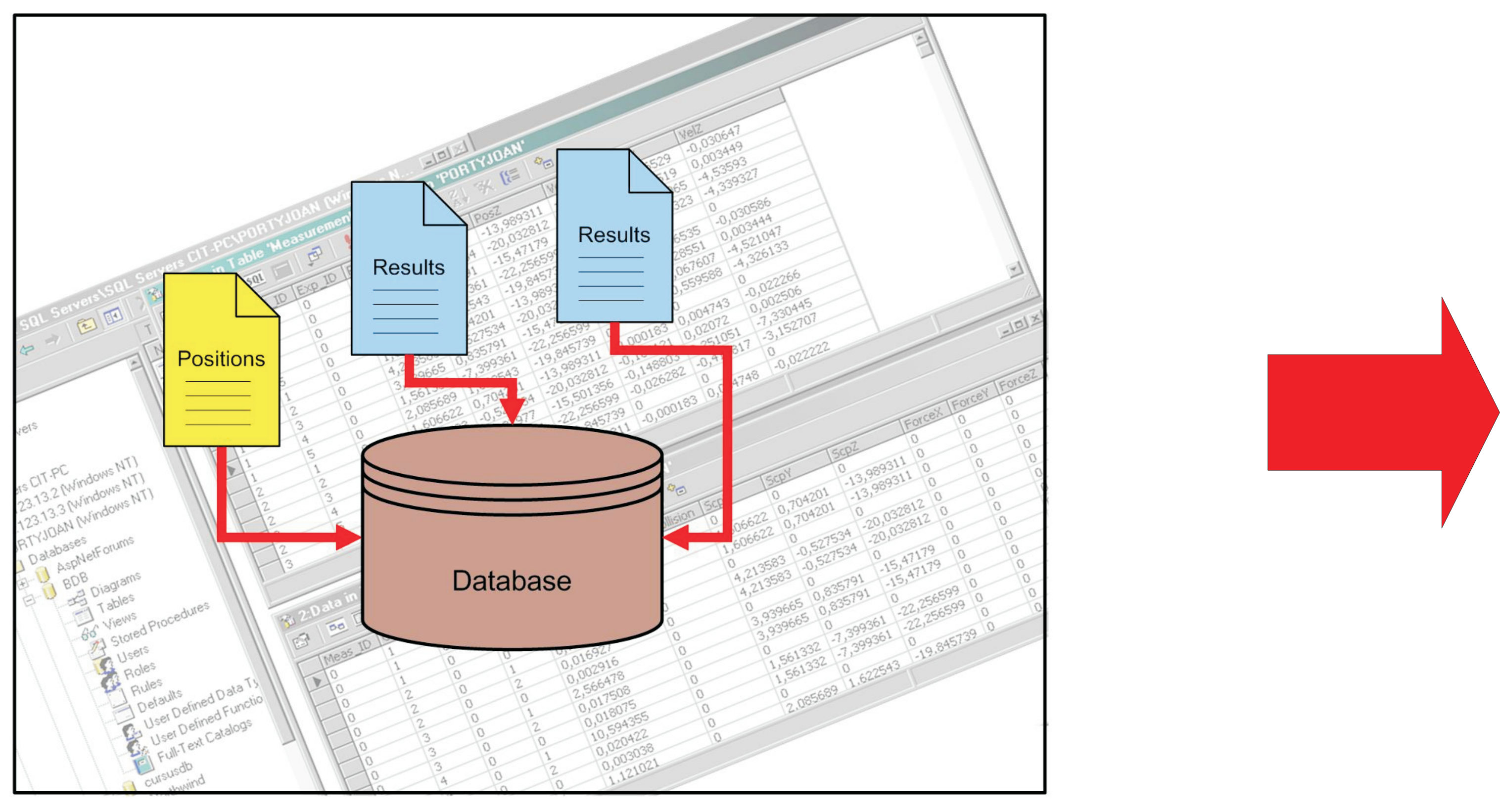

\section{Store Data in Database \\ Since each exploration of a single object by a single user for just a few seconds already results in several thousands of data points, all the result files and the positions file are stored to a database. This allows us to easily query and select data from this huge amount of data.}
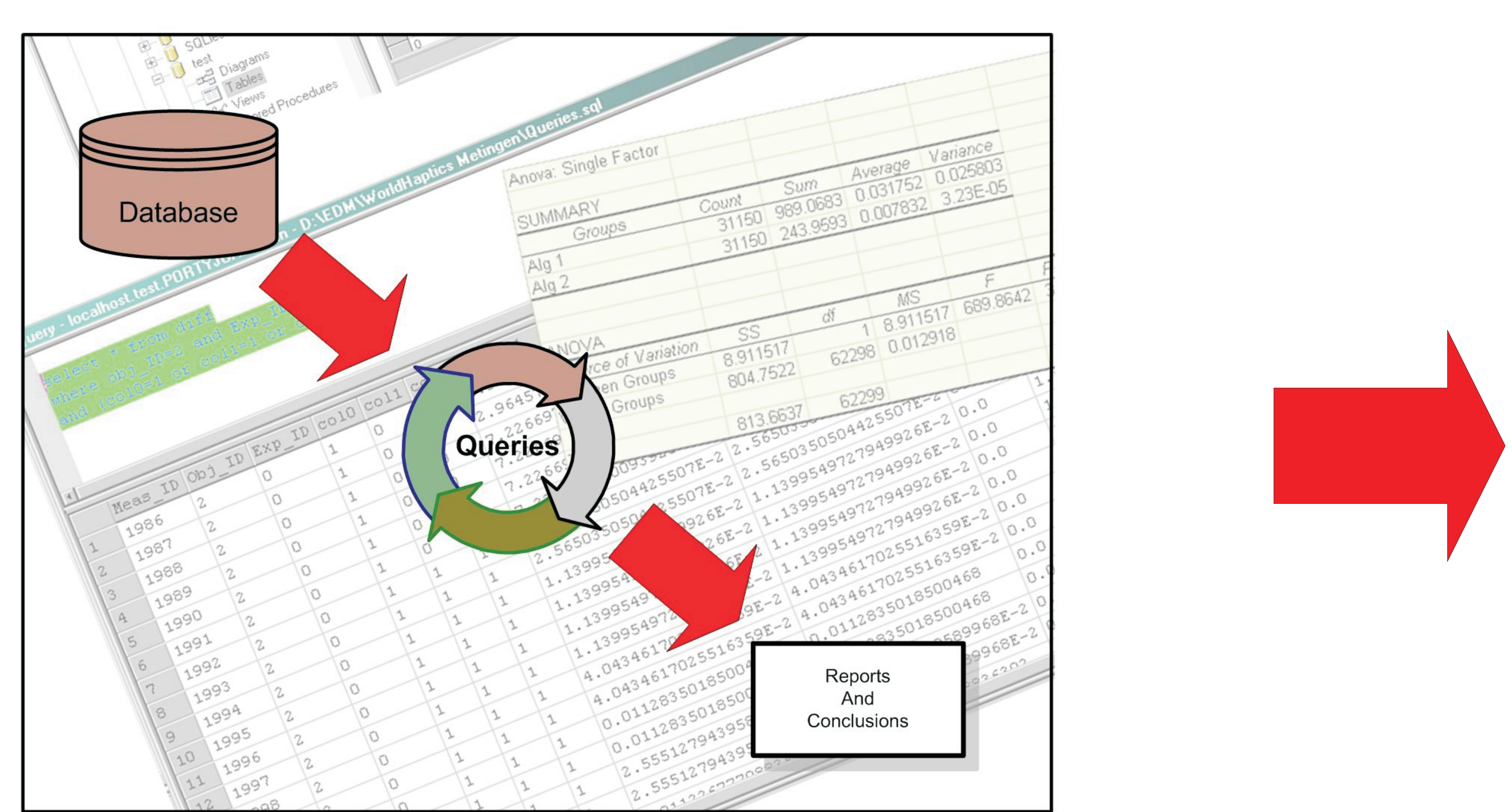

Apply Numerical/Statistical Operations

The conclusions about the correctness and performance of the algorithms, can be drawn by analyzing the data. This can be done directly using the build-in database support, or by exporting a selection to a statistical appliction. 